\title{
Effects of Curved Periacetabular Osteotomy on the Stress Fields of the Pubic Rami and Ischium: A Finite Element Model Analysis
}

\author{
Norio Imai $^{{ }^{*}}$, Yoichiro Dohmae ${ }^{1}$, Ken Suda ${ }^{2}$, Dai Miyasaka ${ }^{2}$, Tomoyuki Ito $^{2}$, Naoto Endo ${ }^{2}$ \\ ${ }^{1}$ Department of Orthopedic Surgery, Niigata Prefectural Shibata Hospital, Niigata, Japan; ${ }^{2}$ Department of Orthopedic Surgery, Nii- \\ gata University Medical and Dental Hospital, Niigata, Japan. \\ Email: *imainorio2001@yahoo.co.jp
}

Received May $18^{\text {th }}, 2013$; revised June $19^{\text {th }}, 2013$; accepted July $1^{\text {st }}, 2013$

Copyright (C) 2013 Norio Imai et al. This is an open access article distributed under the Creative Commons Attribution License, which permits unrestricted use, distribution, and reproduction in any medium, provided the original work is properly cited.

\begin{abstract}
Background: Postoperative pubic or ischial stress fracture may be a complication after curved periacetabular osteotomy (CPO). The discontinuity of the superior pubic rami is a risk factor for this complication. We investigated the stress field differences in standing and sitting positions after CPO. Methods: We used finite element analysis to assess the effects of inferior pubic rami and ischial fractures with or without discontinuity of superior pubic rami. We used the "union model", obtained from a bony union at the osteotomy site of the superior pubic rami from 38-year-old woman who had undergone CPO for left hip dysplasia. We deleted the bony union region and created a discontinuity in the superior pubic rami equal to the non-union, creating the "discontinuity model". We compared the stress field and stress value in the simulated standing and half weight-bearing positions on the operative side, one-legged standing position on the non-operative side, and the sitting position. Findings: In 4 cases, the inferior rami experienced the highest stress. Stress values in the discontinuity model were higher than those in the union model: 1.7 times in the case of one-legged standing on the operative side, 2.4 times in the case of half weight-bearing on the operative side, 3.8 times in the case of one-legged standing on the non-operative side, and 2.0 times in the sitting position, respectively. Interpretation: We recommend patients delay weight bearing on the operative side, avoid the sitting position as long as possible, and sit down slowly to prevent inferior pubic rami and ischial fractures after CPO.
\end{abstract}

Keywords: Curved Periacetabular Osteotomy; Ischial Fracture; Inferior Pubic Ramifracture; Stress Field; Finite Element Analysis

\section{Introduction}

Acetabular osteotomies are useful not only for increasing the covering of the femoral head, but also for medialization of the femoral head. Therefore, they are considered a useful treatment for hip dysplasia in young patients [1-4]. However, the method requires detachment of the gluteus medius muscle; consequently, the surgery is significantly invasive. For this reason, curved periacetabular osteotomy (CPO) [4], considered less invasive than other acetabular osteotomies, was adopted as the treatment of first choice for hip dysplasia in young patients in our institution. However, due to the technical difficulty of these procedures, various complications have been noted, such as collapse of rotational fragment, heterotopic ossifica-

${ }^{*}$ Corresponding author. tion, and infection [5-8].

Postoperative pubic or ischial stress fracture has also been referred as a complication in some reports, and discontinuity of superior pubic rami $[9,10]$ and narrowing of the inferior pubic rami $[10,11]$ have been considered as risk factors. However, a detailed analysis with regard to load distribution after CPO has not been reported. Moreover, we employ the same rehabilitation program for patients at risk as for those who do not exhibit the risk factors, because the fractures do not significantly influence walking or hospital stay $[9,10]$.

The purpose of this study was to investigate stress field differences in standing and sitting positions after CPO. We used computer simulation with finite element analysis to assess effects of inferior pubic rami and ischial fracture with or without discontinuity of superior 
pubic rami. Moreover, we provide recommendations concerning the rehabilitation program of patients with discontinuity of the superior pubic rami.

\section{Materials and Methods}

\subsection{Subject}

The model pelvis and proximal femur were obtained from a 38-year-old woman who had undergone CPO for left hip dysplasia and who underwent a computed tomography (CT) examination 3 months after surgery, when the bony union was still not complete. In these patients, bony union was observed at the osteotomy site of the superior pubic rami (Figure 1). The Institutional Review Board of Niigata Prefectural Shibata Hospital approved the study and informed consent was obtained from the patient.

\subsection{Geometry and Material Properties}

Geometrical data were obtained using a high-definition three-dimensional digitizer (Mimics, Materialise Japan Co. Ltd, Yokohama, Japan). After digitizing the pelvis, the coordinates were imported into a commercially available finite element modeling software package (SIMULIA Abaqus FEA, IDAJ Co. Ltd, Yokohama, Japan) for three-dimensional finite mesh reconstruction. The model was divided into a cortical bone and a cancellous bone, consisting of approximately 800,000 elements and 200,000 nodes. The material properties were as follows: Young's modulus and Poisson's ratio were 17,000 MPa and 0.3, respectively, in cortical bone and $70 \mathrm{MPa}$ and 0.2 , respectively, in cancellous bone [12].

\subsection{Loading and Boundary Conditions}

In the standing position, the anterior pelvic plane, consisting of the bilateral anterior superior iliac spine and

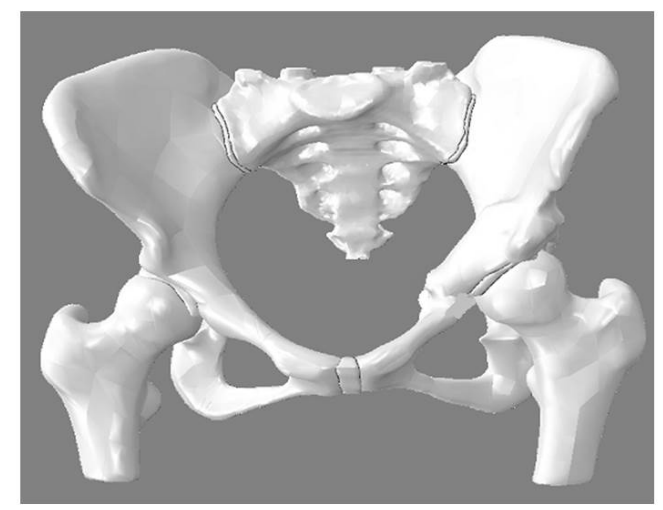

Figure 1. Union model. Pelvic model after CPO on the left side. In this model, bony union was obtained at osteotomy site of superior pubic rami. pubic symphysis, was parallel to vertical, and the superior endplate of the sacrum and non-weighted femur were rigidly fixed. We applied a force of $435 \mathrm{~N}$ for one-legged standing position and $218 \mathrm{~N}$ for half weight bearing, respectively, to the femur from distal. These values were calculated based on the weight of one leg, approximately $15 \%$ of a total weight (preoperative body weight) of 50 $\mathrm{kg}$ [10]. The friction factor of the hip joint was assumed to be 0.01 [12]. In the sitting position, the anterior pelvic plane was tilted $25^{\circ}$ posteriorly, based on a previous report [13], and the superior endplate of the sacrum was rigidly fixed. We applied a force of $174 \mathrm{~N}$ from the most distal point of each bilateral ischium. This value was calculated based on the weight of two legs and a total weight of $50 \mathrm{~kg}(348 \mathrm{~N})$; each leg received half the force $(174 \mathrm{~N})$.

Our study model was originally obtained from a bony union at the osteotomy site of the superior pubic rami (Figure 1); this is defined as the "union model". We deleted the bony union region similar to the osteotomy line by a length of $5 \mathrm{~mm}$, and created a discontinuity in the superior pubic rami equal to non-union; this is defined as the "discontinuity model" (Figure 2). Subsequently, we investigated the difference of load distribution between these 2 models.

\section{Results}

In all 4 cases the inferior rami experienced the highest stress (Figures 3-6).

In the case of one-legged standing position, in the discontinuity model, the stress field $>5.0 \mathrm{MPa}$ was distributed from the inferior rami to the ischium. By contrast, in the union model, the stress field $>5.0 \mathrm{MPa}$ was not observed (Figure 3). Similarly, in the discontinuity model, the stress field >2.5 MPa was distributed from the inferior rami to the ischium, whereas, in the union models,

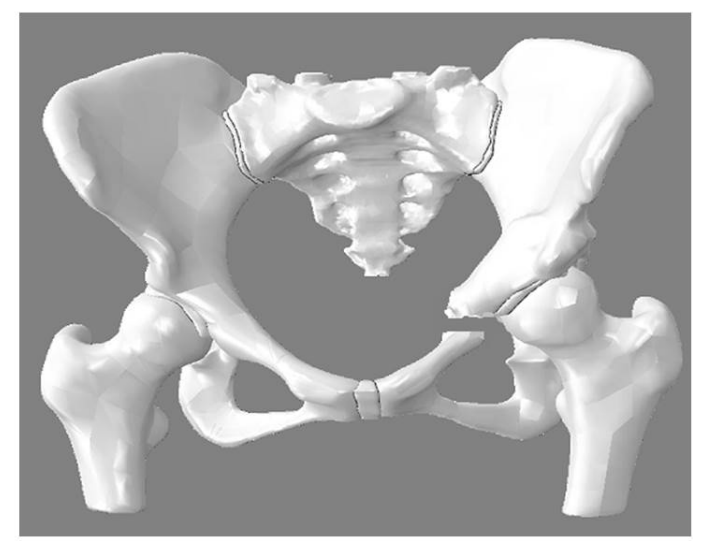

Figure 2. Discontinuity model. In this model, we partially deleted the bony union and created a discontinuity at osteotomy site of superior pubic rami. 


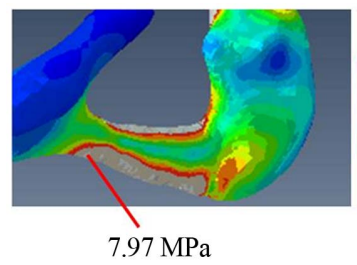

(a)

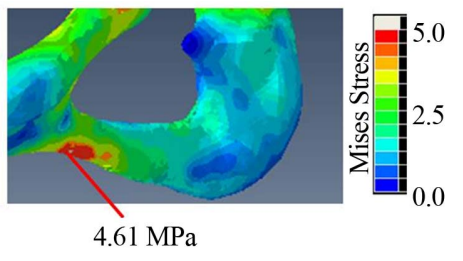

(b)
Figure 3. One-legged standing on the operative side. The highest load was 7.97 MPa in discontinuity model (a) and 4.61 MPa in union model (b).

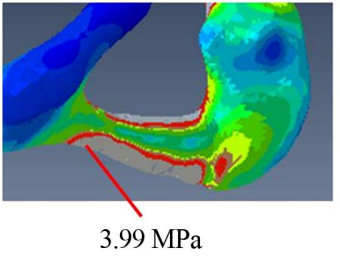

(a)

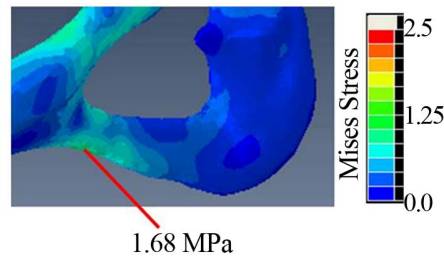

(b)
Figure 4. Half weight bearing on the operative side. The highest load was 3.99 MPa in discontinuity model (a) and 1.68 MPa in union model (b).

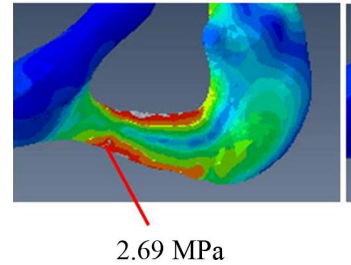

(a)

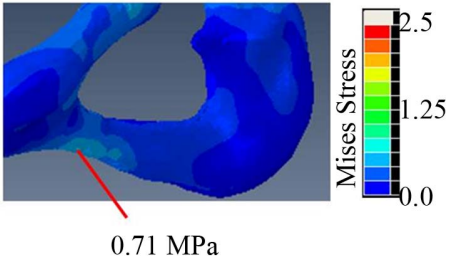

(b)
Figure 5. One-legged standing on the non-operative side. The highest load was 2.69 MPa in discontinuity model (a) and 0.71 MPa in union model (b).

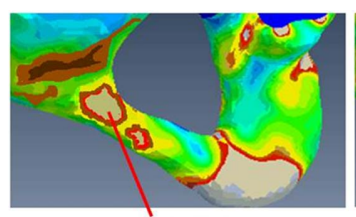

8.47 MPa

(a)

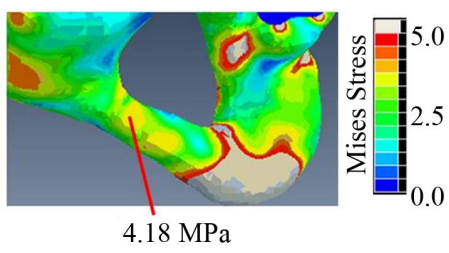

(b)
Figure 6. Sitting position. The highest load was 8.47 MPa in discontinuity model (a) and 4.18 MPa in union model (b).

the stress field $>2.5 \mathrm{MPa}$ was not observed in cases of half weight-bearing on the operative side and one-legged standing on the non-operative side (Figures 4 and 5). In case of sitting position, in the discontinuity models, the stress field >5.0 MPa was observed at two places in the inferior rami. In the union models, the stress field $>5.0$ MPa was not observed, but stress field was higher than others, similar to that in the discontinuity model (Figure 6).
Moreover, stress values in the discontinuity model were higher than those in the union model: 1.73 times higher in the case of one-legged standing on the operative side (7.97 MPa vs. 4.61 MPa in average; Figure 3), 2.38 times higher in the case of half weight bearing on the operative side (3.99 MPa vs. 1.68 MPa in average; Figure 4), 3.79 times higher in the case of one leg standing on the non-operative side (2.69 MPa vs. 0.71 MPa in average; Figure 5), and 2.02 times higher in the sitting position (8.47 MPa vs. 4.18 MPa in average; Figure 6).

\section{Discussion}

Stress fractures in the pubic and ischial bones are relatively rare, and published reports have mostly concentrated the sports-related injuries in young patients [14-16]. A few reports have described the pubic or ischial fracture as a complication of acetabular osteotomies, including CPO [9-11]. In these reports, fractures of the inferior pubic rami and ischial fractures were observed in approximately $3 \%$ - $20 \%$ of patients who had undergone acetabular osteotomies [9-11].

With regard to etiological factors for these fractures, [11] demonstrated that cases with a discontinuity at the site of the superior pubic osteotomy accounted for as many as $80 \%$ of all cases with inferior pubic or ischial fractures, a statistically significant increase compared to cases without pubic or ischial fracture (4.5\%). They inferred that the fractures derive from disruption of continuity at the site of pubic osteotomy, which alters load transfer from the lower extremities, based on past reports $[17,18]$. Our model demonstrates that with discontinuity at the site of osteotomy of the superior pubic rami, the stress field is concentrated from the inferior pubic rami to ischial bone, especially on the proximal inferior pubic rami. These results are consistent with the hypothesis of Tsuboi et al. Postoperative inferior pubic rami fractures and ischial fractures are relatively rare and do not affect the clinical outcome; only $0.3 \%$ of cases required further surgical treatment for painful non-unions. Therefore, most surgeons do not change the rehabilitation schedule, because there is no adequate treatment to help prevent these fractures if a discontinuity at the osteotomy site is observed. In our institution, patients are permitted to use a wheelchair 2 days after surgery. Ten-kilogram partial weight bearing is permitted with 2 crutches after 2 weeks. Subsequently, an increase in weight bearing of $10 \mathrm{~kg}$ per week is permitted, and crutches are removed between 3 and 6 months after the operation if the patient can stand on one leg.

According to several reports [9-11], most fractures occurred as early as 3 months after surgery. The osteotomy site of the inferior rami and ischium may become stronger with a gradually increasing load over a long 
period of time. For a patient with discontinuity at the osteotomy site, we suggest that weight bearing in the operative side be delayed, and we especially recommend non-weight bearing on the operative side. Moreover, we also recommend that patients avoid the sitting position for as long as possible, and take care to sit down slowly.

A limitation of this study is the examination of the only one pelvic model. The load distribution may be different from other pelvic models because of variation in pelvic morphology, especially in the width of the inferior pubic rami or in bone mineral density. Moreover, the discontinuity model in this current study was reconstructed by virtual deletion and not by using an actual CT scan from a patient before healing of superior pubic rami. However, if the model was used before healing of superior pubic rami, other osteotomy sites, such as ilium around the acetabulum will not join, therefore, the distribution may be different. Furthermore, the deletion width in this study was defined as $5 \mathrm{~mm}$, but the actual width will be irregular. Therefore, the stress locations or value may be affected by the deletion width. However, the location of the stress field was similar to that in our previous study [10] in a different pelvic model; therefore, the results of this current study are valid at least with regard to the stress field. Furthermore, in this study, we applied a force of $174 \mathrm{~N}$ from most distal point of each bilateral ischium in the sitting model. There are many muscles and soft tissues around the buttocks, so the load distribution of the inferior pubic rami and ischium may be smaller in actuality.

In conclusion, we demonstrated that discontinuity at the osteotomy site of the superior pubic rami increased the load on the inferior pubic rami and ischium on the osteotomy side. Surgeons should be aware of the fact that these fractures are complications that occur at a relatively early stage after acetabular osteotomies, including CPO. Furthermore, we recommend that weight bearing on the operative side be delayed, and that patients avoid the sitting position for as long as possible, taking care to sit down slowly to prevent inferior pubic rami and ischial fractures after acetabular osteotomies.

\section{REFERENCES}

[1] S. Ninomiya and H. Tagawa, "Rotational Acetabular Osteotomy for the Dysplastic Hip," The Journal of Bone and Joint Surgery. American Volume, Vol. 66, No. 3, 1984, pp. 430-436.

[2] R. Ganz, K. Klaue, T. S. Vinh and J. W. Mast, “A New Periacetabular Osteotomy for the Treatment of Hip Dysplasias. Technique and Preliminary Results,” Clinical Orthopaedics and Related Research, Vol. 7, No. 232, 1988, pp. 26-36.

[3] Y. Hasegawa, T. Iwase, S. Kitamura, K. Yamauchi, S.
Sakano and H. Iwata, "Eccentric Rotational Acetabular Osteotomy for Acetabular Dysplasia: Follow-Up of One Hundred and Thirty-Two Hips for Five to Ten Years," The Journal of Bone and Joint Surgery. American Volume, Vol. 84, No. 3, 2002, pp. 404-410.

[4] M. Naito, K. Shiramizu, T. Akiyoshi, M. Ezoe and Y. Nakamura, "Curved Periacetabular Osteotomy for Treatment of Dysplastic Hip,” Clinical Orthopaedics and Related Research, Vol. 4, No. 433, 2005, pp. 129-135. doi:10.1097/01.blo.0000153281.75265.1d

[5] J. Crockarell Jr., R. T. Trousdale, M. E. Cabanela and D. J. Berry, "Early Experience and Results with the Periacetabular Osteotomy. The Mayo Clinic Experience," Clinical Orthopaedics and Related Research, Vol. 7, No. 363, 1999, pp. 45-53.

[6] J. P. Davey and R. F. Santore, "Complications of Periacetabular Osteotomy,” Clinical Orthopaedics and Related Research, Vol. 6, No. 363, 1999, pp. 33-37.

[7] R. Biedermann, L. Donnan, A. Gabriel, R. Wachter, M. Krismer and H. Behensky, "Complications and Patient Satisfaction after Periacetabular Pelvic Osteotomy,” International Orthopaedics, Vol. 32, No. 5, 2008, pp. 611617. doi:10.1007/s00264-007-0372-3

[8] J. C. Clohisy, A. L. Schutz, L. St. John, P. L. Schoenecker and R. W. Wright, "Periacetabular Osteotomy: A Systematic Literature Review,” Clinical Orthopaedics and Related Research, Vol. 467, No. 8, 2009, pp. 2041-2052. doi:10.1007/s11999-009-0842-6

[9] N. Espinosa, J. Strassberg, E. L. Belzile, M. B. Millis and Y. J. Kim, "Extraarticular Fractures after Periacetabular Osteotomy,” Clinical Orthopaedics and Related Research, Vol. 466, No. 7, 2008, pp. 1645-1651. doi:10.1007/s11999-008-0280-X

[10] N. I. Mai, Y. Dohmae, K. Suda, D. Miyasaka, T. Ito and N. Endo, "The Finite Element Analysis for Pubic Rami and Ischium Fracture after or during Curved Periacetabular Osteotomy,” Hip Joint, Vol. 12, 2012, pp. 571-574.

[11] M. Tsuboi, Y. Hasegawa, K. Fujita and K. Kawabe, "Pubic/Ischial Stress Fractures after Eccentric Rotational Acetabular Osteotomy,” Journal of Orthopaedic Science, Vol. 16, No. 1, 2011, pp. 38-43. doi:10.1007/s00776-010-0010-6

[12] Z. Li, N. B. Butala, B. S. Etheridge, H. J. Siegel, J. E. Lemons and A. W. Eberhardt, "A Biomechanical Study of Periacetabular Defects and Cement Filling,” Journal of Biomechanical Engineering, Vol. 129, No. 2, 2007, pp. 129-136. doi:10.1115/1.2472367

[13] S. Nishihara, N. Sugano, T. Nishii, K. Ohzono and H. Yoshikawa, "Measurements of Pelvic Flexion Angle Using Three-Dimensional Computed Tomography,” Clinical Orthopaedics and Related Research, Vol. 6, No. 411, 2003, pp. 140-151.

doi:10.1097/01.blo.0000069891.31220.fd

[14] C. Smets, J. Roos, E. Vanlommel and T. D. Bunker, "Stress Fracture of Os Ischium,” Injury, Vol. 26, No. 6, 1995, pp. 411-412. doi:10.1016/0020-1383(95)00044-A

[15] P. F. Hill, S. Chatterji, D. Chambers and J. D. Keeling, 
"Stress Fracture of the Pubic Ramus in Female Recruits," The Journal of Bone \& Joint Surgery, Vol. 78, No. 3, 1996, pp. 383-386.

[16] T. R. Williams, M. L. Puckett, G. Denison, A. Y. Shin and J. D. Gorman, “Acetabular Stress Fractures in Military Endurance Athletes and Recruits: Incidence and MRI and Scintigraphic Findings,” Skeletal Radiology, Vol. 31, No. 5, 2002, pp. 277-281. doi:10.1007/s00256-002-0485-0
[17] M. Dalstra and R. Huiskes, "Load Transfer across the Pelvic Bone,” Journal of Biomechanics, Vol. 28, No. 6, 1995, pp. 715-724. doi:10.1016/0021-9290(94)00125-N

[18] N. Kaku, H. Tsumura, H. Taira, T. Sawatari and T. Torisu, "Biomechanical Study of Load Transfer of the Pubic Ramus Due to Pelvic Inclination after Hip Joint Surgery Using a Three-Dimensional Finite Element Model," Journal of Orthopaedic Science, Vol. 9, No. 3, 2004, pp. 264-269. doi:10.1007/s00776-004-0772-9 